\title{
Rising Mean Platelet Volume (MPV) Heralding Platelets Recovery in Dengue?
}

\author{
Ajay Khandal ${ }^{1, *}$, D. Raghuraman ${ }^{2}$ \\ ${ }^{1}$ Department of Medicine, Prathima Institute of Medical Sciences, Karimnagar, India \\ ${ }^{2}$ Chairman Institutional ethics committee, Prathima Institute of Medical Sciences, Karimnagar, India \\ *Corresponding author:drkhandal@gmail.com
}

\begin{abstract}
Infectious diseases including Dengue illness remains the commonest cause of hospitalization in Indian settings. It is a significant global public health problem, with 2.5 billion world's population at risk and an estimated 50 to 390 million annual infections. Clinically, dengue illness manifestation varies from asymptomatic state to severe symptomatic disease. Symptomatic disease events in turn range from self-limiting dengue fever (DF) to severe dengue. Severe dengue, manifests as; dengue hemorrhagic fever (DHF), hypovolemic shock-dengue shock syndrome (DSS) or severe organ impairment. Thrombocytopenia is a common element to both dengue fever and severe dengue; and clinicians, unfortunately, tend to 'react' to thrombocytopenia with platelet transfusions at predetermined platelet thresholds. Readily available lab parameters to guide the clinician of impending platelet recovery is needed, one such parameter gaining recent interest is Platelet Indices (PIs): mean platelet volume (MPV) and platelet distribution width (PDW). Serially observing mean platelet volume along with Platelets might be valuable, with a rising trend in MPV suggesting platelet and patient recovery.
\end{abstract}

Keywords: dengue, thrombocytopenia, dengue cost of hospitalization, thrombocytopenia mechanisms

Cite This Article: Ajay Khandal, and D. Raghuraman, "Rising Mean Platelet Volume (MPV) Heralding Platelets Recovery in Dengue?" American Journal of Clinical Medicine Research, vol. 5, no. 4 (2017): 59-63. doi: 10.12691/ajcmr-5-4-5.

\section{Introduction}

Infectious diseases including dengue illness are by far the most common reason for hospitalization in Indian settings - representing around $25 \%$ of all admissions. In fact, infectious diseases as a cause of hospitalization are more common than, cardiovascular, respiratory, neurology, hematology, endocrine, and cancer-related etiology combined. [1] Dengue illness remains a significant clinical and public health challenge globally; with 2.5 billion world's population at risk and an estimated 50 to 390 million annual infections. [2] It is an arboviral disease transmitted by Aedes aegypti and Aedes albopictus mosquitoes, and is one of the most important mosquitoborne viral illness. Clinically, dengue illness manifestation varies from asymptomatic to severe symptomatic disease. Pathologically, bleeding events is consequent to microangiopathy and thrombocytopenia; shock due to capillary plasma leakage; and organ failure state representing a combination of shock or microangiopathy associated hypoxic damage. Thrombocytopenia is a common element to both dengue fever and severe dengue.

The platelet counts are now done routinely by automated/ semi-automated cell counters. In practice, appropriate use of all parameters reported by cell counters in the complete blood count (CBC) report is lacking. [3,4] Among the parameters provided, platelet indices are probably the most 'ignored' by clinical laboratories and not reported routinely. [4] Moreover, lack of awareness of its role in various disease course among physicians allows persistence of such practices by clinical laboratories, as physicians in practice, hardly send a requisition for the comprehensive report. Additionally, methodological issues and standardization difficulties mandate each laboratory determine its reference range, [4] as different hematological cell counter use results in differing values. [5] The Platelet Indices (PIs) are believed to have a role in predicting the clinical course in various disease states: from infectious, inflammatory, metabolic to even acute abdomen; and in many of these conditions, platelets are presumed to play a pathogenic role. [6-10] Furthermore, PIs are considered valuable in suggesting the pathophysiologic mechanism of thrombocytopenia. [11] Also, in dengue it is believed to have value in diagnosing and classifying the disease into Dengue Fever (DF) and Severe Dengue. [12,13].

Private healthcare facilities are the primary source of medical care in India; with well over $70 \%$ spell of illness treated by them in both rural and urban areas $(72 \%$ in rural and 79\% in urban). [1] Most of such expenditure is out of pocket--as health insurance penetration is negligible in both urban and rural households; and families incur the expenses by utilizing household savings or borrowing, adding a significant financial burden to the economically marginalized sections. [1] Furthermore, 'financial constraints' (57\% in rural, $68 \%$ in urban) remained the reason for the inability on the part of patients to access healthcare. [1] The average cost to treat dengue on the outpatient basis is estimated at $\$ 23.49$ and $\$ 26.09$ respectively, depending on 
the treatment taken at a public or a private sector. Conversely, hospitalized patients incur ten times the cost, which does not differ significantly between public or private for-profit setups (\$197.03 vs. \$248.11) [2,14,15].

The notable financial burden during the management of dengue fever is admission cost, as approximately $82 \%$ of the expenditure incurred in dengue is due to hospitalization. $[2,14]$ Conversely, around two-third patients of dengue, were managed at one-fifth of cost when managed on an ambulatory basis. [2] Furthermore, the significant chunk of hospital expense is resultant to platelet transfusion, as a single transfusion cost around Rs.12,000 (\$200); in fact, the economic burden of 2006 Dengue epidemic in Delhi is an estimated, \$27.4 million. [16] Clinicians in practice have a tendency to 'chase platelets' with consequent multiple transfusions at predefined platelet thresholds (triggers), such behavior on the part of clinicians is reinforced by patients family members, and is curiously termed: "The Dengue Panic Syndrome." [17] Lab parameters to guide the clinician of an impending thrombocytopenia recovery is urgently needed; as it can result in significant out-of-pocket saving; one such recent parameter reported recently is Immature platelet fraction (IPF) estimation, which predicts platelet recovery and outcome. [18] Unfortunately it is not available with all semi-automatic analyzers, the ones used commonly in resource-limited rural setups. Conversely, the readily available PIs: Mean Platelet Volume (MPV), Platelet Distribution Width (PDW), reported by most automatic and semi-automatic cell counters may serve as a useful guide. The below described two cases address the role of PIs (MPV) in the prediction of platelet recovery in dengue if any.

\section{Case 1}

A 31-year-old female with dengue fever, hypotension, and thrombocytopenia (Severe Dengue) was referred from a nearby village for platelet transfusion. She was managed on lines of 'typhoid-malaria' since last one week by community-level informal practitioners many of whom lack any formal diploma/ degree in health care. Later the patient was investigated with necessary lab tests routinely as she reported 'not feeling well' with persistent nausea and vomiting (Dengue warning signs). The platelets were found to be alarmingly low-19,000; dengue nonstructural protein 1 (NS1) antigen, rapid card test, was positive; which led to her immediate referral. At presentation she complained of vaginal bleeding, without any petechia or tourniquet test positivity, she had her last cycle 12 days back and had no history of irregular menstruation. On examination: the patient was afebrile, her Pulse was 98/minute, Blood Pressure (BP) was 96/80 mm Hg, blood oxygen saturation level (SpO2) -98 on ambient air, she was not in any distress, the systemic examination was normal. The labs revealed: Haemoglobin $(\mathrm{Hb})-13.0 \mathrm{~g} / \mathrm{dl}$, hematocrit (HCT)-39\%, platelet count $-19,000 / \mu 1$, total

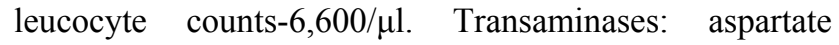
transaminase (AST) and alanine transaminase (ALT) were raised mildly, at 56 and 60 IU respectively; other routine parameters viz., electrolytes, renal function was normal.
Chest Xray Posteroanterior-view (CXR-PA View) and USG abdomen did not show any evidence of pleural fluid or ascites. Complete urine examination (CUE), prothrombin time (PT) and activated partial thromboplastin time (aPTT) were all normal. Dengue Rapid Diagnostic Kit Test (Jay Mitra) was positive for NS1 antigen and IgM; the IgG was nonreactive.

She was started on fluids and other supportive measures. Taking into account, vaginal bleeding, Pulse Pressure of 16 (BP being 96/80), and severe thrombocytopenia (platelets $<20,000 / \mu \mathrm{l})$ consideration for Platelet transfusion was made--as further precipitous fall in platelets, might result in clinical deterioration. But was deferred, the financial constraints on the part of the family and otherwise clinical stability of the patient added to the decision process.

The possibility of capillary leakage and consequent clinical deterioration after fluid infusions guided her close monitoring. Her general condition improved with the simple measure of hydration, her Blood Pressure improved to $110 / 80 \mathrm{~mm} \mathrm{Hg}$ by the second day of therapy, transaminases normalized by the third day, and her vaginal bleeding stopped by the fourth. She made an uneventful recovery and was discharged with a platelet count greater than 50,000/ $\mathrm{ll}$. During the hospitalization platelet indices (PIs): mean platelet volume (MPV); platelet distribution width (PDW); and platelet counts, were serially observed (Table 1); and found to be statistically significant.

Table 1.

\begin{tabular}{|c|c|c|c|}
\hline $\begin{array}{c}\text { DAY OF } \\
\text { HOSPITALISATION }\end{array}$ & Platelet count & $\begin{array}{c}\text { Mean platelet } \\
\text { volume } \\
\text { (MPV) }\end{array}$ & $\begin{array}{c}\text { Platelet } \\
\text { distribution } \\
\text { width (PDW) }\end{array}$ \\
\hline DAY 1 & $\mathbf{2 6 0 0 0}$ & 9 & 14 \\
\hline DAY 2 & $\mathbf{2 9 0 0 0}$ & 10.1 & 18.2 \\
\hline DAY 3 & $\mathbf{3 1 0 0 0}$ & 11.8 & 22.4 \\
\hline DAY 4 & $\mathbf{4 0 0 0 0}$ & 11.1 & 26.6 \\
\hline DAY 5 & $\mathbf{6 1 0 0 0}$ & 14.2 & 30.8 \\
\hline
\end{tabular}

Pearson Correlation Coefficient (R) is 0.9068 ; the P-Value is 0.033674 . The result is significant at $\mathrm{p}<0.05$.

Platelet counts per micrometer (uL) of blood; MPV in femtoliter (fL); PDW in percentage (\%).

\section{Case 2}

A 19-year old male, presented with fever with chills, headache, myalgia, nausea, over the ensuing four days; on examination was found to have: temperature $102 * \mathrm{~F}$, hypotension (Blood pressure: 90/60 $\mathrm{mm} \mathrm{Hg}$ ), tourniquet test was in the negative; his systemic examination was noncontributory. Lab studies revealed: thrombocytopenia (platelets: 66,000/uL), NS1 reactive. In consideration of dengue warning signs (persistent vomiting), the patient was initiated on home based fluids. He had few episodes of vomiting the following day, but his general condition gradually improved with simple measures of fluids and general care. His platelet counts and platelet indices were observed serially over the ensuing four days and are tabulated (Table 2), but was not found to be statistically significant. 
Table 2.

\begin{tabular}{|c|c|c|c|}
\hline $\begin{array}{c}\text { Day of } \\
\text { Fever }\end{array}$ & $\begin{array}{c}\text { Platelet } \\
\text { Counts }\end{array}$ & $\begin{array}{c}\text { Mean Platelet } \\
\text { Volume (MPV) }\end{array}$ & $\begin{array}{c}\text { Platelet distribution } \\
\text { width (PDW) }\end{array}$ \\
\hline DAY 4 & 66000 & 10.9 & 15.3 \\
\hline DAY 5 & 52000 & 10.9 & 15.1 \\
\hline DAY 6 & 66000 & 10.8 & 15.6 \\
\hline DAY 7 & 48000 & 10.9 & 15.3 \\
\hline DAY 8 & 61000 & 11.3 & 15.7 \\
\hline DAY 9 & 79000 & 11.2 & 15.4 \\
\hline
\end{tabular}

Pearson Correlation Coefficient (R) is: 0.3868 ; the P-Value is 0.448735 . The result is not significant at $\mathrm{p}<0.05$.

Platelet counts per micrometer (uL) of blood; MPV in femtoliter (fL); PDW in percentage (\%).

\section{Discussion}

A trend of considering most acute undifferentiated fevers (AUFs) as malaria or typhoid is rampant in limited resource settings including India. The above consideration leads to over usage of antimalarials and enteric lines of antibiotics; consequent to such an approach, there is widespread resistance now seen to chloroquine in malaria and fluoroquinolones in the case of enteric fever. $[19,20]$ Despite the changed view of World Health Organisation (WHO) to not use antimalarials indiscriminately without evidence of disease either by microscopy or antigen-based rapid diagnostic test (RDTs), [21] its usage continues unabated. Adding credence to such an approach is recent PCR-based studies that have demonstrated a high prevalence of malaria ranging from (6-35\%) in AUFs, along with limitations of microscopy and RDTs in diagnosis. [22] Compared to PCR, microscopy had a sensitivity of $29 \%$ and specificity of $98 \%$, while the RDT had a sensitivity of $24 \%$ and specificity of $99 \%$. [22] Such differences in evidence compounded with the highly varied prevalence of diseases between regions or between localities in the same area, creates an unusual challenge for the treating physician during decision making in AUF.

The first case (reported partially earlier), [23] depicts all the challenges discussed above regarding AUFs; the patient's treatment on lines of enteric fever and malaria, for a prolonged period without considering other aetiologies being one. Secondly, the warning signs of severe dengue (persistent nausea and vomiting) did not alert the health care provider to find an alternate diagnosis of fever. As, the village level care for illness is not straightforward, and on a majority of occasions such care is provided by informal health providers (who lack any formal education in health) due to the lack of appropriately trained medical faculty. Moreover, a fever is considered a simple ailment unworthy of specialized attention. [24]

Clinically, dengue illness presents with nonspecific symptoms, but certain features can help to make a provisional diagnosis. WHO includes Fever and two of the following criteria to diagnose probable dengue: nausea, vomiting; rash; aches and pains; tourniquet test positive; leucopenia (WBC $<5,000 / \mathrm{ul}$ ) and associated warning signs. The latter include abdominal pain or tenderness; persistent vomiting; clinical fluid accumulation like polyserositis; mucosal bleed; lethargy or restlessness; liver enlargement $>2 \mathrm{~cm}$; increase in HCT concurrent with rapid decrease in platelet count. [25] The patients with dengue warning signs require strict observation and medical intervention. [25] Severe dengue is a manifestation of profound plasma leakage resulting in the following: one, shock (Dengue Shock Syndrome-DSS). Two, third-space fluid loss. Three, severe bleeding as evaluated by a clinician. Four, severe organ involvement like liver, (AST or ALT $>=1000$ ), central nervous system (Impaired consciousness), or other vital organs like the heart. [25] The lack of field knowledge on these factors result in significant delay in diagnostic stratification and appropriate simple measures of fluid resuscitation as seen in case 1.

PIs role in the prediction of hemorrhagic diathesis in thrombocytopenia is suggested since the early 80 's. [26] However various studies of PIs in dengue lack uniformity, probably due to methodological issues and standardization difficulties, in measuring PIs. [4,5]. As different autoanalyzer can have different values, making it difficult to compare between studies, necessitating each study define its mean [27-30]. In Dengue illness an MPV of less than nine has been suggested to have a diagnostic potential with a high reported sensitivity. [12,31] Hence, in the case 1. The first MPV report of nine could have provided a clue for dengue illness. Secondly, the continued observation of MPV over the ensuing four days, in case 1 , suggests a recovery pattern which is found to be statistically significant (Table 1). Studies on PIs correlation with platelets from the beginning of the illness to recovery lacks in dengue, consequently available literature is not clear in regards to the association or otherwise between PIs and platelet recovery, some studies which have observed the patients for three days found it to be unrelated. [34]. However, a trend of correlation strength over the three days in their study is hard to miss (R-0.159 on day 1; R- 0.214 day 2; R-0.45 day 3). [32] In case 2, the patient was followed similar to case 1, but was found to have a modest association, which was statistically insignificant (Table 2). But, the case 2 was studied on a different autoanalyzer, and importantly the patient could not be followed till his complete recovery. Nonetheless, a similar lack of association has been reported by other studies. [32,33]

Part of this asymmetric results in dengue also lies with the pathogenesis of thrombocytopenia, which is not entirely known, with two primary hypotheses. First, decreased production resultant to bone marrow depression by dengue virus. Second, immune (anti-dengue antibodies/ immune complex) mediated destruction of platelets, or peripheral sequestration. [34] In general low MPV, is seen with the former mechanisms and higher MPV is seen with the latter. In Indian circumstance, peripheral destruction has been suggested to be the predominant mechanism in more than half of patients. [35] Thus, in the setting of a low MPV with low platelets, a rising trend in MPV may herald platelet recovery as the thrombocytopenia in these subsets is resultant to bone marrow depression. Conversely, a persistently raised MPV along with ongoing thrombocytopenia implies peripheral (immune mediated) destruction, and in these subsets, an increased MPV suggests the strength of inflammation and resultant platelet destruction, rather than recovery. [36] The case 1 was probably representative of marrow suppression, in 
case 2. The mechanism of thrombocytopenia was unclear as the MPV remained at more or less the same level during observation and was non-indicative of either a marrow suppression or peripheral destruction.

In hospitalized patients with dengue, Platelet transfusion tendency at predetermined set points (triggers) is to be discouraged, as no credible evidence exists to such an approach. [37,38] Moreover, it may cause significant harm as a novel mechanism of pathogenesis in dengue suggests a role of von Willebrand factor (vWF) and its cleaving protease ADAMTS13 (a disintegrin-like and metalloprotease with thrombospondin type 1 domain 13) imbalance as a putative mechanism. [39] In dengue, the damage to the endothelium results in the release of $\mathrm{vWF}$, which in such settings (inflammation) is ultra-large vWF (UL-vWF). UL-vWF binds to the platelets avidly and forms microthrombi. ADAMTS13 cleaves the UL-vWF in healthy individuals and prevents the formation of such micro plugs and consequent vasculopathy (microangiopathy). [40] As dengue illness can result in an excess of UL-vWF and deficiency of ADAMTS13, the transfusion of platelets alone without replacement of ADAMTS13 may lead to further platelet plugs and microangiopathy. Or in other words, platelet transfusion may cause more harm than good and add insult to the ongoing injury. Consequently, transfusion of fresh frozen plasma (FFP) which theoretically replaces the ADAMTS13 protease has been suggested to have a beneficial role in dengue. [41] WHO suggests a role of fresh whole blood transfusion in dengue, which provides both platelets and ADAMTS13 protease. [23] Furthermore, intravenous immunoglobulin and plasma exchange as a modality of therapy is representative of such improved understanding of dengue illness. [42,43] The case 1 was managed conservatively despite, having severe thrombocytopenia with simple measures and watchful waiting, and calls for a thoughtful approach towards such patient given the new clinicopathologic understanding. In case 2 , early recognition of dengue warning signs leads to timely intervention with fluids and recovery.

\section{Conclusion}

Dengue is a common illness with significant morbidity, understanding the pathophysiology allows one to make better therapeutic choices. Low MPV with low platelets implies marrow suppression as a mechanism of thrombocytopenia. High MPV with ongoing thrombocytopenia represents peripheral destruction and needs other therapeutic options beyond the typical platelet transfusions. In such settings, an easily available fresh whole blood transfusion or FFP may be more rewarding clinically. In marrow depression as the cause of thrombocytopenia, a rising trend in MPV heralds platelet recovery, and a wait-full watch might well suffice. Such an approach can result in significant decline in dengue expense burden.

\section{References}

[1] Government of India (2015) Key Indicators of Social Consumption in India Health. NSS 71Round. January - June 2014. Ministry of Statistics and Programme Implementation. National Sample Survey Office.
[2] Shepard DS, Halasa YA, Tyagi BK, et al. Economic and Disease Burden of Dengue Illness in India. The American Journal of Tropical Medicine and Hygiene. 2014; 91(6): 1235-1242.

[3] Ayalew et al.: How to Interpret and Pursue an Abnormal Complete Blood Cell Count in Adults, Mayo Clin Proc. 2005;80(7): 923-936.

[4] Farias, M., Schunck, E., Dal Bó, S., et al. (2009). Definition of reference ranges for the platelet distribution width (PDW): a local need. Clinical Chemistry and Laboratory Medicine, 48(2), pp. 255-257.

[5] Lippi G, Pavesi F, Pipitone S. Evaluation of mean plate- let volume with four haematological analyzers: har- monization is still an unresolved issue. Blood Coagul Fi- brinolysis 2015; 26: 235-7.

[6] Larsen SB, Grove EL, Hvas AM, Kristensen SD. Platelet turnover in stable coronary artery disease-influence of thrombopoietin and low-grade inflammation. PLoS One. 2014; 9: e85566.

[7] Ates I, Bulut M, Ozkayar N, Dede F. Association between high platelet indices and proteinuria in patients with hypertension. Ann Lab Med. 2015; 35: 630-4.

[8] Öztürk ZA, Dag MS, Kuyumcu ME, Cam H, Yesil Y, Yilmaz N, et al. Could platelet indices be new biomarkers for inflammatory bowel diseases? Eur Rev Med Pharmacol Sci. 2013; 17: 334-41.

[9] Budak YU, Polat M, Huysal K. The use of platelet indices, plateletcrit, mean platelet volume and platelet distribution width in emergency non-traumatic abdominal surgery: a systematic review. Biochemia Medica. 2016; 26(2): 178-193.

[10] Murat Afyon et al.. Could mean platelet volume be a useful marker for infectious diseases? a review of literature, medscience. 2016. 05. 8460.

[11] Katti TV, Mhetre SC, Annigeri C. How far are the platelet indices mirror image of mechanism of thrombocytopenia-mystery still remains? Int J Adv Med 2014; 1: 200-5.

[12] Bashir AB, Saeed OK, Mohammed BA, Ageep AK. Role of Platelet Indices in Patients with Dengue Infection in Red Sea State, Sudan. IJSR. 2015; 4(1): 1573-6.

[13] Dewi YP. Mean Platelet Volume (MPV): Potential Predictor of Disease Severity in Dengue infection. In proceeding of: International Dengue Symposium. 2013 conference paper.

[14] Economic and disease burden of dengue in Southeast Asia. Shepard DS, Undurraga EA, Halasa YA. PLoS Negl Trop Dis. 2013; 7(2): e2055.

[15] National Vector Borne Disease Control Programme. Dengue Cases and Deaths in the Country since 2007. Ministry of Health and Family Welfare, Directorate General of Health Services; 2013.

[16] Garg P, Nagpal J, Khairnar P, Seneviratne SL. Economic burden of dengue infections in India. Trans R Soc Trop Med Hyg. 2008; 102: 570-7.

[17] Ahluwalia G, Sharma SK. Dengue: Current Trends and Challenges - An Indian Perspective. J Assoc Physicians India. 2004; 52: 561-3.

[18] Dadu, T., Sehgal, K., Joshi, M. and Khodaiji, S. (2014), Evaluation of the immature platelet fraction as an indicator of platelet recovery in dengue patients. Int. Jnl. Lab. Hem., 36: 499-504.

[19] Rajnish Joshi, John M. Colford Jr., Arthur L. Reingold, and Shriprakash Kalantri* Nonmalarial Acute Undifferentiated Fever in a Rural Hospital in Central India: Diagnostic Uncertainty and Overtreatment with Antimalarial Agents Am. J. Trop. Med. Hyg., 78(3), 2008, pp. 393-399.

[20] Araya Gebreyesus wasihun, Letemichael Negash Wlekidan, Senay Aregawi Gebremariam, Abadi Luel Welderufael, Saravanan Muthupandian, Tadesse Dejenie Haile, Tsehaye Asmelash Dejene, Diagnosis and Treatment of Typhoid Fever and Associated Prevailing Drug Resistance in Northern Ethiopia, International Journal of Infectious Diseases, Volume 35, 2015, Pages 96-102.

[21] Guidelines for the treatment of malaria - 3rd edition. World Health Organization 2015.

[22] Haanshuus CG, Chandy S, Manoharan A, Vivek R, Mathai D, Xena D, et al. (2016) A High Malaria Prevalence Identified by PCR among Patients with Acute Undifferentiated Fever in India. PLoS ONE 11(7): e0158816.

[23] Handbook for clinical management of dengue. World Health Organization 2012.

[24] Khandal A. MPV and DENGUE. IJIRAS.Volume 4 Issue 6, June 2017.

[25] Runi Datta. The World of Quacks: A Parallel Health Care System in Rural West Bengal IOSR Journal Of Humanities And Social Science (IOSR-JHSS). 
[26] Eldor A, Avitzour M, OR R, Hanna R, Penchas S. Prediction of haemorrhagic diathesis in thrombocytopenia by mean platelet volume. Br Med J. 1982; 285(6339): 397-400.

[27] Demirin H, Ozhan H, Ucgun T, Celer A, Bulur S, Cil H, et al. Normal range of mean platelet volume in healthy subjects: insight from a large epidemiologic study. Thromb Res. 2011; 128 : 358-60.

[28] Sachdev R, Tiwari AK, Goel S, Raina V, Sethi M. Establishing biological reference intervals for novel platelet parameters (immature platelet fraction, high immature platelet fraction, platelet distribution width, platelet large cell ratio, platelet-X, plateletcrit, and platelet distribution width) and their correlations among each other. Indian J Pathol Microbiol. 2014; 57: 231-5.

[29] Maluf CB, Barreto SM, Vidigal PG. Standardization and reference intervals of platelet volume indices: Insight from the Brazilian longitudinal study of adult health (ELSA-BRASIL). Platelets. 2015; 26: 413-20.

[30] Lippi G, Pavesi F, Pipitone S. Evaluation of mean platelet volume with four haematological analyzers: harmonization is still an unresolved issue. Blood Coagul Fibrinolysis. 2015; 26: 235-7.

[31] Navya BN, Patil S, Kariappa TM. Role of platelet parameters in dengue positive cases - an observational study. Int J Health Sci Res. 2016; 6(6):74-78.

[32] Prakash GM, Anikethana G V. Use of mean platelet volume and platelet distribution width in predicting trend in platelet count and bleeding risks in patients of dengue fever. Int J Adv Med 2016; 3 : 611-3.

[33] Wiwanitkit V. Mean platelet volume in the patients with dengue hemorrhagic fever, Platelets 2004;15(3):185.

[34] Elzinandes Leal de Azeredo, Robson Q. Monteiro, and Luzia Maria de-Oliveira Pinto, "Thrombocytopenia in Dengue: Interrelationship between Virus and the Imbalance between Coagulation and Fibrinolysis and Inflammatory Mediators," Mediators of Inflammation, vol. 2015, Article ID 313842, 16 pages, 2015.
[35] KRISHNAMURTHY, Vani; RAJESHAKAR, Rajalakshmi; DORESWAMY, Srinivasa Murthy. Thrombocytopenia in Dengue illness: Destruction, Suppression and Composite platelet index: A Retrospective study. Annals of Pathology and Laboratory Medicine, [S.1.], v. 3, n. 5, p. A465-470, nov. 2016.

[36] Khaleed JK, Abeer Anwer AA, Maysem Alwash AA. Platelet indices and their relations to platelet count in hypoproductive and hyper-destructive Thrombocytopenia. Karbala J. Med. 2014; 7: 1952-8.

[37] David C. Lye, Vernon J. Lee, Yan Sun, Yee Sin Leo; Lack of Efficacy of Prophylactic Platelet Transfusion for Severe Thrombocytopenia in Adults with Acute Uncomplicated Dengue Infection. Clin Infect Dis 2009; 48 (9): 1262-1265.

[38] Preventive transfusion in dengue shock syndrome-is it necessary? Lum, Lucy Chai See et al.The Journal of Pediatrics , Volume 143, Issue 5, 682-684.

[39] Rossi, F. C. et al., Transfusion, A novel association of acquired ADAMTS13 inhibitor and acute dengue virus infection 2010, 50(1), 208-212.

[40] Djamiatun K, van der Ven AJAM, de Groot PG, Faradz SMH, Hapsari D, Dolmans WMV, et al. (2012). Severe Dengue Is Associated with Consumption of von Willebrand Factor and Its Cleaving Enzyme ADAMTS-13. PLoS Negl Trop Dis 6(5): e1628.

[41] Sellahewa, K. H., Samaraweera, N., Thu- sita, K. P. and Fernando, J. L., Is fresh frozen plasma effective for thrombocytopenia in adults with dengue fever? A prospective randomised double blind controlled study. Ceylon Med. J., 2008, 53(2), 36-40.

[42] Castro RA, Castro JA, Barez MY, Frias MV, Dixit J, Genereux M. Thrombocytopenia associated with dengue hemorrhagic fever responds to intravenous administration of anti - D $(\mathrm{Rh}(\mathrm{O})-\mathrm{D})$ immune globulin. Am J Trop Med Hyg. 2007;76(4):737-742.

[43] Pannu AK, Bhalla A, Singhal M, Suri V, Sha q N, Varma S. Safety and ef cacy of a single dose of Anti-D (WinRho ${ }^{\circledR}$ ) in severe thrombocytopenia secondary to dengue virus infection. Indian J Crit Care Med 2017; 21: 80-4. 